\title{
Optical Properties of Solar Absorbers - Results on Round Robin and Guidelines
}

\author{
M.João Carvalho', Soraia Páscoa ${ }^{1}$, Fabienne Sallaberry ${ }^{2}$, Helen Rose Wilson ${ }^{3}$, Stephan \\ Fischer $^{4}$, Anders Loren ${ }^{5}$, Andreas Bohren ${ }^{6}$ and Jan Erik Nielsen ${ }^{7}$ \\ ${ }^{1}$ LNEG - Laboratório Nacional de Energia e Geologia, Lisboa (Portugal) \\ ${ }^{2}$ CENER - Centro Nacional de Energías Renovables, Sarriguren (Spain) \\ ${ }^{3}$ Fraunhofer Institute for Solar Energy Systems ISE, Freiburg (Germany) \\ ${ }^{4}$ ITW, Stuttgart (Germany) \\ ${ }^{5}$ RISE - Research Institutes of Sweden, Borås (Sweden) \\ ${ }^{6}$ SPF - Institut für Solartechnik, Hochschule für Technik, Rapperswil (Switzerland) \\ ${ }^{7}$ Solarkey, Hvalsoe (Denmark)
}

\begin{abstract}
The scope of the standard ISO 22975-3:2014 is the determination of the long term behavior and service life of selective solar absorbers for use in vented flat plate collectors used in typical solar domestic hot water systems or combisystems. With this scope, this standard can be the basis for the certification of solar absorbers in the framework of Solar Keymark European Certification Scheme. Measurements of optical properties are fundamental to support this certification. Six test laboratories participated in a Round Robin test for the measurement of solar absorptance and thermal emittance of different absorber coatings. The samples used were one PVD coating and two selective paint coatings all on aluminum substrates.

The results were submitted anonymously and were analyzed according to standard ISO 13528:2015. The results obtained showed good agreement among the measurements made by the participants and allowed the preparation of guidelines to guarantee quality of the measurements of optical properties of solar absorbers.
\end{abstract}

A proposal to include absorber coatings in the Solar Keymark certification scheme was prepared.

Keywords: Absorber Coatings, Optical Properties, Round Robin

\section{Introduction}

In the framework of the international standardization committee ISO TC 180, standards for collector components and materials are being developed. The first standard published is ISO 22975-3:2014. The scope of this standard covers the determination of the long-term behavior and service life of selective solar absorbers for use in vented flat plate collectors used in typical solar domestic hot water systems or combisystems. This standard can be the basis for the certification of solar absorber coatings in the framework of the Solar Keymark Certification Scheme.

ISO 22975-3:2014 uses a failure criterion based on the optical properties for the evaluation of the durability of the absorbers. The measurement of the optical properties, namely solar absorptance and thermal emittance, is to be performed according to Annex A of ISO 22975-3:2014. In order to evaluate the quality of the measurements performed according to this standard, six laboratories cooperated to perform a Round RobinRound Robin for the measurement of these parameters. 


\section{Methodology}

\subsection{Round Robin Procedure}

The main purpose of this Round Robin was to compare the solar absorptance and thermal emittance measurements of absorber coatings for flat plate collectors. The Round Robin procedure was agreed among the six test laboratories and based on Annex A of ISO 22975-3:2014. Six samples of three different selective coatings (one PVD and two paint coatings) were distributed to the participating laboratories and two measurement rounds were performed. Each laboratory measured three samples during the first round, then sent those three samples to the next laboratory and finally measured the next three samples that it had received from another laboratory during the second round.

Results were submitted anonymously to the evaluator. The standard ISO 13528:2015 was used to analyze the results of the Round Robin.
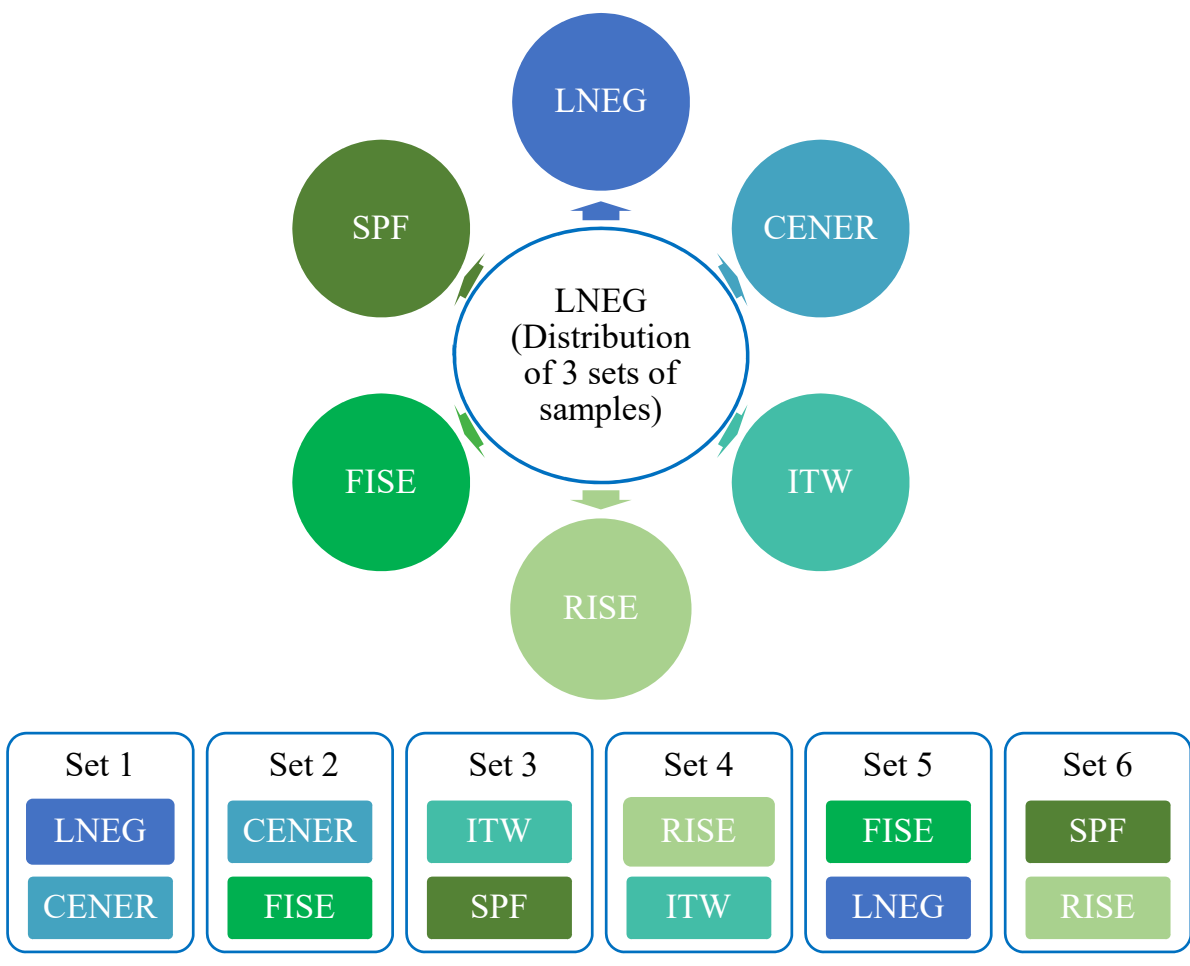

Fig. 1 - Samples distribution and its exchange between laboratories.

\subsection{Evaluation of spectral absorptance values and spectral emittance values}

According to Annex A of ISO 22975-3:2014, the evaluation of the solar absorptance $(\alpha)$ is based on the measurement of spectral near-normal/hemispherical reflectance of the sample, $R_{s}(\lambda)$ and measurement of spectral near-normal/hemispherical reflectance of the reference, $R_{r}(\lambda)$. The spectral range for this measurement is from $0.30 \mu \mathrm{m}$ to $2.50 \mu \mathrm{m}$, with at least 40 wavelengths measured. The calculation of near-normal/hemispherical spectral absorptance $\alpha(\lambda)$ is given by:

$$
\alpha(\lambda)=1-\rho(\lambda)=1-\frac{R_{s}(\lambda)}{R_{r}(\lambda)} \rho_{r}(\lambda)
$$

where: $\rho_{r}(\lambda)$ is the near-normal/ hemispherical reflectance of the reference.

According to Annex A from ISO 22975-3 (2014), the evaluation of the thermal emittance $\varepsilon(T)$ is also based on the measurement of spectral near-normal/hemispherical reflectance of the sample, $R_{s}(\lambda)$ and measurement of spectral near-normal/hemispherical reflectance of the reference, $R_{r}(\lambda)$.

The spectral range for this measurement is from $2.0 \mu \mathrm{m}$ to $50.0 \mu \mathrm{m}$. If only a narrower wavelength range is available for measurements (e.g. up to $25 \mu \mathrm{m}$ ), the last measured value can be considered as constant up to $50 \mu \mathrm{m}$ 
for the following calculations.

The calculation of near-normal/hemispherical spectral emittance $\varepsilon(T)$ is given by:

$$
\varepsilon(\lambda)=\mathbf{1}-\boldsymbol{\rho}(\lambda)=\mathbf{1}-\frac{R_{s}(\lambda)}{R_{r}(\lambda)} \rho_{r}(\lambda)
$$

where: $\rho_{r}(\lambda)$ is the near-normal/ hemispherical reflectance of the reference.

\subsection{Determination of solar absorptance and thermal emittance of absorber surfaces}

According to Annex A from ISO 22975-3:2014, the solar absorptance $\alpha_{\mathrm{s}}$ is calculated from the following expression:

$$
\boldsymbol{\alpha}_{s}=\frac{\sum_{i=1}^{n}\left[\alpha\left(\lambda_{\mathrm{i}}\right) \times S_{\lambda_{\mathrm{i}}} \times \Delta \lambda_{\mathrm{i}}\right]}{\sum_{i=1}^{n}\left[S_{\lambda_{\mathrm{i}}} \times \Delta \lambda_{\mathrm{i}}\right]}
$$

where

$\alpha(\lambda)$ is the near-normal/hemispherical spectral absorptance as defined in Equation 1

$S_{\lambda_{\mathrm{i}}}$ is the reference solar spectral irradiance (ISO 9050:2003)

$\lambda_{\mathrm{i}}$ is the selected measuring wavelength

$\Delta \lambda_{\mathrm{i}}$ is the respective wavelength interval

The thermal emittance $\varepsilon(T)$ is calculated from the following expression:

$$
\varepsilon(T)=\frac{\sum_{i}^{n} \varepsilon\left(\lambda_{\mathrm{i}}\right) \times P_{\lambda_{\mathrm{i}}}(T) \times \Delta \lambda_{\mathrm{i}}}{\sum_{i}^{n} \boldsymbol{P}_{\lambda_{\mathrm{i}}}(T) \times \Delta \lambda_{\mathrm{i}}}
$$

where

$P_{\lambda_{\mathrm{i}}}(T)$ is the value of the Planck function for black body radiation at the selected wavelengths and temperature

$\varepsilon(\lambda)$ is the near-normal/hemispherical spectral emittance as defined in Equation 2

$\lambda_{\mathrm{i}}$ is the selected measuring wavelengh

$\Delta \lambda_{\mathrm{i}}$ is the respective wavelength interval

The Planck function is defined by the following expression:

$$
E(\lambda, T)=\frac{c_{1}}{\lambda^{5}\left[\exp \left(\frac{c_{2}}{\lambda T}\right)-1\right]}
$$

where

$C_{1}=8 \pi h c\left[\mathrm{Wm}^{2}\right]=3.74 \times 10^{-16}\left[\mathrm{Wm}^{2}\right]$

$C_{2}=\frac{h c}{k}[\mathrm{mK}]=1.44 \times 10^{-2}[\mathrm{mK}]$

and

$E$ is the spectral radiance $\left(\mathrm{Js}^{-1} \cdot \mathrm{m}^{-2} \cdot \mathrm{sr}^{-1} \cdot \mathrm{Hz}^{-1}\right)$

$T$ is the temperature of the black body $(\mathrm{K})$

$h$ is Planck's constant (Js)

$c$ is the velocity of light $\left(\mathrm{ms}^{-1}\right)$

$e$ is Euler's number (dimensionless) 


\subsection{Analyses of Round Robin results}

The analyses of the Round Robin results was performed according to standard ISO 13528: 2015.

For the calculation of the standard deviation for proficiency assessment (Round Robinn), $\sigma_{\mathrm{pt}}$, the normalized interquartile range was considered, as seen in Eq. 6. This is considered as a robust estimator of the standard deviation of the participant results.

$$
\sigma_{p t}=n I Q R(x)=0.7413\left[Q_{3}(x)-Q_{1}(x)\right]
$$

where:

$\mathrm{Q}_{1}(\mathrm{x})$ is the 25 th percentile of $\mathrm{xi}(\mathrm{i}=1, \ldots, 6)$

$\mathrm{Q}_{3}(\mathrm{x})$ is the 75 th percentile of $\mathrm{xi}(\mathrm{i}=1, \ldots, 6)$

A correction for non-homogeneity of the distributed samples was introduced by considering the estimator of standard deviation given by Eq. 7:

$$
\sigma_{p t}^{\prime}=\sqrt{\sigma_{p t}^{2}+s_{s}^{2}}
$$

where $\mathrm{s}_{\mathrm{s}}$ is the "between samples" standard deviation as defined in ISO 13528:2015. The comparison of the results was made using the z-score value defined by:

$$
z_{i}=\frac{\left(x_{i}-x_{p t}\right)}{\sigma^{\prime} p t}
$$

where:

$\mathrm{x}_{\mathrm{pt}}$ is the assigned value and $\mathrm{x}_{\mathrm{i}}$ the measured value.

The assigned value of the proficiency test (Round Robin) is the median of the values measured by each participant. The interpretation of z-scores according to ISO 13528:2015 is:

- A result that gives $|z| \leq 2.0$ is considered to be satisfactory;

- A result that gives $2.0 \leq|z| \leq 3.0$ is considered to be questionable;

- A result that gives $|z| \geq 3.0$ is considered unsatisfactory.

\section{Results and discussion}

To check the homogeneity of the test items (samples distributed), a measurement of the optical properties of all samples was made by LNEG before the distribution of samples.

According to ISO 22975-2 the test samples to be used in the durability tests shall have standard deviation in the determined values of solar absorptance of less than 0.01 and for the determined values of thermal emittance of less than 0.04 .

The sets of three samples, each with six specimens, distributed and initially measured by LNEG showed the following standard deviations (table 1), which satisfy the criteria of ISO 22975-2.

Tab. 1: Values of standard deviation of samples, as determined by a single laboratory

\begin{tabular}{|c|c|c|}
\hline Sample Type & Solar Absorptance & Thermal Emittance \\
\hline Sample A & 0.001 & 0.001 \\
\hline Sample B & 0.001 & 0.008 \\
\hline Sample C & 0.008 & 0.023 \\
\hline
\end{tabular}

As a criterion for homogeneity, the standard ISO 13528:2015 indicates that the "between specimens" standard 
deviation, $s_{s}$, and the standard deviation for proficiency assessment, $\sigma_{\mathrm{pt}}$, shall satisfy Eq. 9:

$$
s_{s} \leq \mathbf{0}, 3 \sigma_{p t}
$$

The calculation of the "between sample" standard deviation was performed according to section B3, of Annex B of the standard and used, for each sample type, three repeated measurements for each of six samples. The number of specimens recommended by ISO 13528:2015 for the homogeneity check is 10 but the Round Robin only has six partners; for this reason, only six specimens of each sample are considered.

For each sample type and for each parameter measured (solar absorptance and thermal emittance), the values of $\mathrm{s}_{\mathrm{s}}$ and $\sigma_{\mathrm{pt}}$ are listed in Table 2. For calculation of $\sigma_{\mathrm{pt}}$, all measurements were considered (first and second round measurement).

Table 2 - Values of $s_{\mathrm{s}}$ and $\sigma_{\mathrm{pt}}$ as determined in the Round Robin with six participating laboratories

\begin{tabular}{|c|c|c|c|l|c|c|c|c|}
\hline & \multicolumn{3}{|c|}{ Solar Absorptance } & \multicolumn{4}{c|}{ Thermal Emittance } \\
\hline & $\mathrm{s}_{\mathrm{s}}$ & $\sigma_{\mathrm{pt}}$ & $0.3 \sigma_{\mathrm{pt}}$ & & $\mathrm{s}_{\mathrm{s}}$ & $\sigma_{\mathrm{pt}}$ & $0.3 \sigma_{\mathrm{pt}}$ & \\
\hline Sample A & 0.0337 & 0.0028 & 0.0008 & NHom & $0^{1}$ & 0.0371 & 0.0111 & Hom \\
\hline Sample B & 0.0337 & 0.0038 & 0.0011 & NHom & 0.0067 & 0.0200 & 0.0060 & NHom \\
\hline Sample C & 0.0923 & 0.0096 & 0.0029 & NHom & 0.0248 & 0.0474 & 0.0142 & NHom \\
\hline
\end{tabular}

From Table 2 we can conclude that for the parameter solar sbsorptance, all sample types have to be considered non-homogeneous (Nhom) and for thermal emittance only sample type A can be considered homogeneous (Hom).

In Table 3, the median values $\mathrm{x}_{\mathrm{pt}}$ of each parameter and sample type are presented as well as the corresponding standard deviation $\sigma_{\mathrm{pt}}$ of the proficiency test and the values corresponding to the z-score limits. Table 4 shows the same values considering the homogeneity correction.

Tab. 3: Compilation of values of median $\mathrm{x}_{\mathrm{pt}}$ and standard deviation $\sigma_{\mathrm{pt}}$ of proficiency test, $\sigma_{\mathrm{pt}}$, without homogeneity correction.

\begin{tabular}{|c|c|c|c|c|c|c|}
\hline Parameter / Sample type & $\mathbf{x p t}$ & $\sigma_{\mathbf{p t}}$ & $\mathbf{Z = - 3}$ & $\mathbf{Z}=\mathbf{- 2}$ & $\mathbf{Z}=\mathbf{2}$ & $\mathbf{Z}=\mathbf{3}$ \\
\hline$\alpha_{\mathrm{s}}$ / Sample A & 0.948 & 0.0009 & 0.945 & 0.946 & 0.950 & 0.951 \\
\hline$\alpha_{\mathrm{s}}$ / Sample B & 0.928 & 0.0023 & 0.921 & 0.923 & 0.933 & 0.935 \\
\hline$\alpha_{\mathrm{s}}$ / Sample C & 0.913 & 0.0069 & 0.892 & 0.899 & 0.926 & 0.933 \\
\hline$\varepsilon(\mathrm{T})$ / Sample A & 0.059 & 0.0371 & $-0.052^{2}$ & $-0.015^{2}$ & 0.133 & 0.170 \\
\hline$\varepsilon(\mathrm{T})$ / Sample B & 0.489 & 0.0200 & 0.429 & 0.449 & 0.529 & 0.549 \\
\hline$\varepsilon(\mathrm{T})$ / Sample C & 0.511 & 0.0474 & 0.369 & 0.416 & 0.606 & 0.653 \\
\hline
\end{tabular}

Tab. 4: Compilation of values of median $\mathrm{x}_{\mathrm{pt}}$ and standard deviation $\sigma_{\mathrm{pt}}$ of proficiency test, $\sigma_{\mathrm{pt}}$, with homogeneity correction.

\begin{tabular}{|c|c|c|c|c|c|c|}
\hline Parameter / Sample type & $\mathbf{x}_{\mathbf{p t}}$ & $\sigma_{\mathbf{p t}}$ & $\mathbf{Z = - 3}$ & $\mathbf{Z}=\mathbf{- 2}$ & $\mathbf{Z = 2}$ & $\mathbf{Z}=\mathbf{3}$ \\
\hline$\alpha_{\mathrm{s}}$ / Sample A & 0.948 & 0.0015 & 0.944 & 0.945 & 0.951 & 0.952 \\
\hline$\alpha_{\mathrm{s}}$ / Sample B & 0.928 & 0.0025 & 0.920 & 0.923 & 0.933 & 0.936 \\
\hline$\alpha_{\mathrm{s}}$ / Sample C & 0.913 & 0.0109 & 0.880 & 0.891 & 0.934 & 0.945 \\
\hline$\varepsilon(\mathrm{T})$ / Sample A & 0.059 & 0.0371 & $-0.052^{2}$ & $-0.015^{2}$ & 0.133 & 0.170 \\
\hline$\varepsilon(\mathrm{T})$ / Sample B & 0.489 & 0.0211 & 0.426 & 0.447 & 0.531 & 0.552 \\
\hline$\varepsilon(\mathrm{T})$ / Sample C & 0.511 & 0.0536 & 0.350 & 0.404 & 0.618 & 0.672 \\
\hline
\end{tabular}

In Figs. 1 to 6 the Z-scores for each sample and parameter are presented considering the homogeneity correction.

\footnotetext{
${ }^{1}$ The value obtained is -4E-06, and for negative values $\mathrm{s}_{\mathrm{s}}$ is considered to be equal to 0 (ISO 13528:2015).

2 This limit is not in agreement with physical considerations since the lower limit value is 0 .
} 
Considering the correction for non-homogeneity, the parameter solar absorptance shows one questionable result for sample A, one questionable result for sample B and for sample $\mathrm{C}$ all results are satisfactory. The results with non-homogeneity correction for the parameter thermal emittance show a questionable result for sample A and for Sample B two unsatisfactory results. For sample C all results are satisfactory. According to the standard ISO 13528:2015, participants should check their measurement procedures in case of questionable or unsatisfactory results.

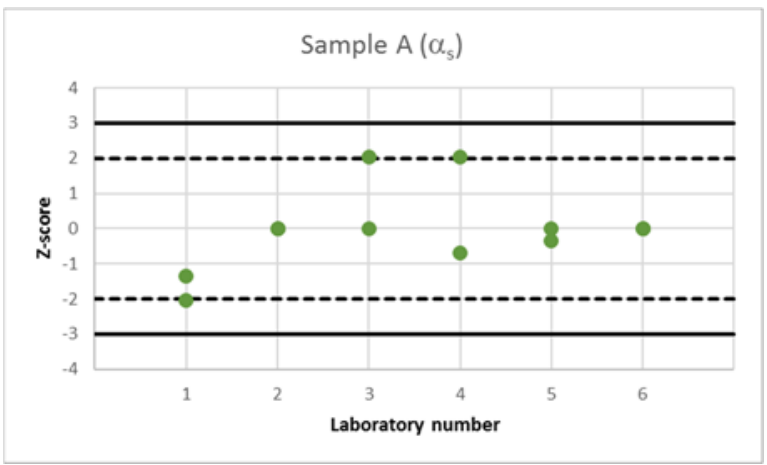

Fig. 1: z-score of parameter $\alpha_{\text {s }}$ for sample type A, with correction for sample inhomogeneity

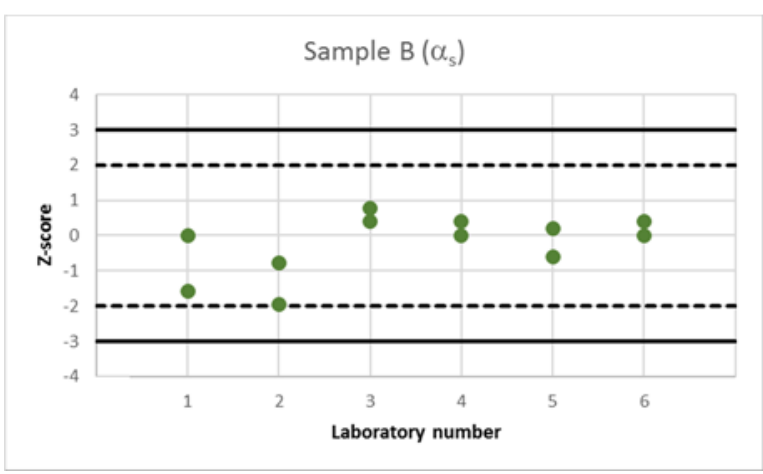

Fig. 3: z-score of parameter $\alpha$ for sample type $B$, with correction for sample inhomogeneity

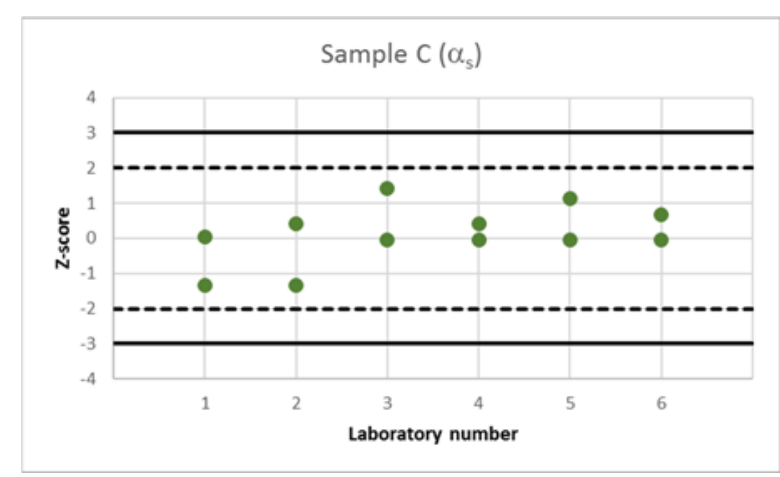

Fig. 5: z-score of parameter $\alpha$ s for sample type C, with correction for sample inhomogeneity

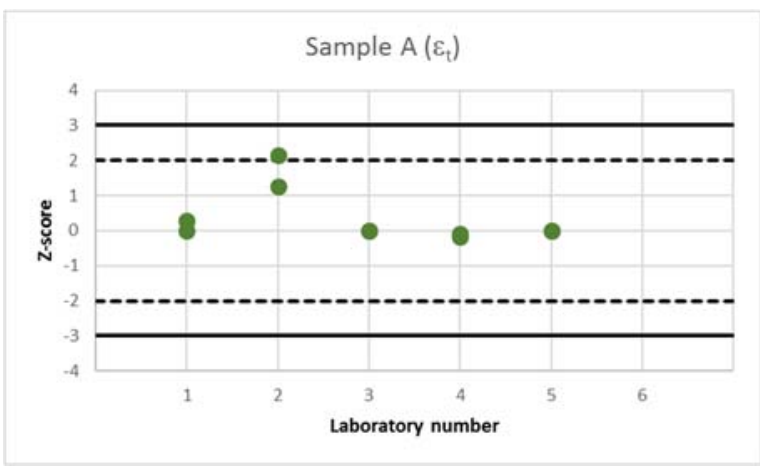

Fig. 2: z-score of parameter $\varepsilon_{t}$ for sample type $A$, with correction for sample inhomogeneity

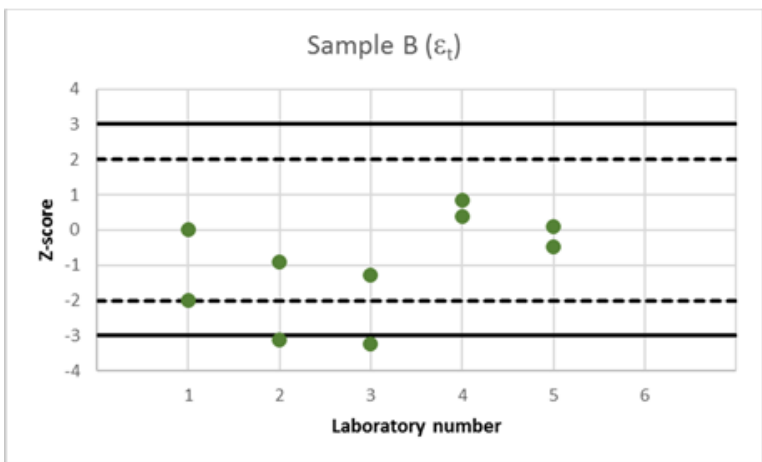

Fig. 4: z-score of parameter $\varepsilon t$ for sample type B, with correction for sample inhomogeneity

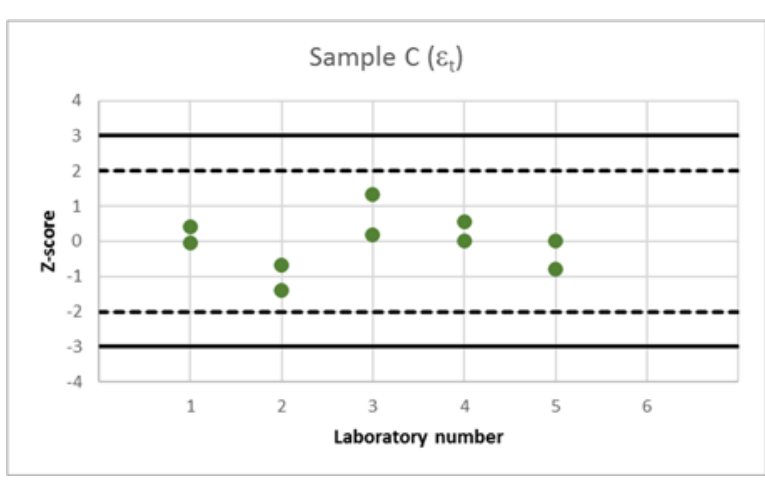

Fig. 6: z-score of parameter $\varepsilon_{t}$ for sample type $C$, with correction for sample inhomogeneity.

\section{Guidelines}

Based on the results of the Round Robin, guidelines were prepared for the determination of the parameters, solar absorptance and thermal emittance,. The guidelines present the necessary equations for calculation of the 
parameters, which are as indicated in section 2. of this article.

Additional aspects to be considered in the measurement are:

a. Samples with a linear structure (typical of rolled metal) should be mounted for be measured according to the schematic representation of Fig. 7. This is to increase the probability of determining an accurate near-normal/hemispherical reflectance value for the spatially inhomogeneous reflection ("streak") in widespread commercial integrating spheres. To evaluate whether this is necessary, photographs with high resolution e.g. using SEM - scanning electron microscope, may be needed, but often the structures are visible to the naked eye.

b. Adequate sampling for representativeness of production line variation but the fulfilling requirements of ISO 22975-3:2014;

c. Correct maintenance of measuring equipment - annual maintenance is a good practice;

d. Traceability of the calibration spectra of reference materials R r $(\lambda)$ used in equations 1 and 2.

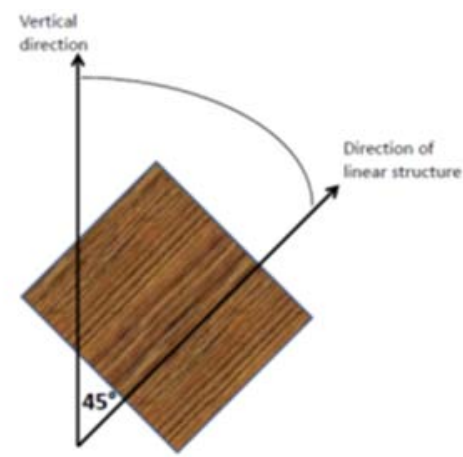

Fig. 7: Visualization of the recommended mounting position, assuming that the optical plane of the spectrophotometer is horizontal

\section{Proposal for Solar Keymark Scheme Rules}

In the framework of the Solar Certification Fund project that supported this work, a proposal for revision of Solar Keymark Scheme rules was prepared to allow the possibility to certify absorber coatings according to ISO 22975 3:2014.

It is considered that the selection of samples for the absorber coating certification shall be done such that it is representative of the whole production (e.g. samples from the left side of the coil, from the middle and from the right side). The test report shall include photos of the samples used in the tests (before and after each test) and the test laboratory shall store samples in good conditions for any necessary future measurements or tests. For instance, the standard conditions for sample conditioning can be considered as $23 \pm 2{ }^{\circ} \mathrm{C}$ and $50 \pm 5 \%$ relative humidity, according to the standard ISO 3270:1984.

\section{Conclusions}

It is possible to conclude that the measurement of solar absorptance and thermal emittance can be done with a majority of satisfactory results by the different test laboratories. Guidelines were prepared that can support other laboratories to perform good quality measurements. For future such interlaboratory comparisons intended to demonstrate measurement proficiency, it is strongly recommended that the homogeneity of specimens for a single sample type is ensured before distribution, so that the effects of sample inhomogeneity do not have to be compensated by additional statistical analysis.

based on the results of the Round Robin a document with guidelines for the measurement of the optical properties 
was prepared. A proposal for a change in the Solar Keymark Scheme rules was considered to allow the certification of absorber coatings for flat plate collectors according to ISO 22975-3:2014.

\section{Acknowledgement}

This work was possible due to the support of the Solar Certification Fund to the project 8C02.1 SK-Abs_LNEG Solar Keymark Scheme rules for absorber coatings - guidelines for comparable optical properties measurements.

This work was also supported in the framework of the project POCI-01-0145-FEDER-016709 (Ref ${ }^{\mathrm{a}}$ FCT PTDC/EMS-ENE/0578/2014) supported by COMPETE 2020 and LISBOA 2020 under the PORTUGAL 2020 Partnership Agreement through the European Regional Development Fund (ERDF) and supported by FCT through National Funds.

\section{References}

ISO 22975-3: 2014 Solar energy - collector components and materials - part 3: Absorber surface durability

ISO 13528: 2015 Statistical methods for use in proficiency testing by interlaboratory comparison.

ISO 9050: 2003, Glass in building - Determination of light transmittance, solar direct transmittance, total solar energy transmittance, ultraviolet transmittance and related glazing factors.

ISO 3270: 1984, Paints and varnishes and their raw materials - Temperatures and humidities for conditioning and testing.

Specific CEN Keymark Scheme Rules for Solar Thermal Products (DRFAT incl. absorber coatings), Version 30 - April 2017

Deliverable D4 - Guidelines - SCF 8th Call - Project: SCF8-SK-Abs - Solar Keymark Scheme rules for absorber coatings - guidelines for comparable optical properties measurements. May 2018 